Revista de Matemática: Teoría y Aplicaciones 2006 13(2) : 95-109

CIMPA - UCR - CCSS ISSN: 1409-2433

\title{
ANILLOS SEPARABLES, DE BAER Y PP-ANILLOS
}

\author{
JoRGe I. GUIER*
}

Recibido/Received: 04/05/06 - Aceptado/Accepted: 08/09/06

\begin{abstract}
Resumen
Keimel introdujo en [5], la noción de proyectabilidad en la clase de anillos reticulados y $f$-anillos. Aquí introducimos una noción similar olvidando la estructura reticular y de orden, e interpretando la perpendicularidad mediante la estructura multiplicativa del anillo (en la clase de anillos reducidos). Esta noción resulta coincidir con la que define la clase de PP-anillos y se relaciona también con la compacidad del espacio de ideales primos minimales y los anillos de Baer débil.
\end{abstract}

Palabras clave: productos booleanos, clases elementales, secciones continuas, anillos de Baer, PP-anillos, ideales primos minimales.

\begin{abstract}
Keimel introduced the notion of proyectability in the class of lattice-ordered rings and $f$-rings in [5]. Here we introduce a similar notion forgeting the lattice and ordered structure of the ring and interpretating the orthogonality within the multiplicative structure (in the class of reduced rings). This notion turns out to be equivalent to the one defining the class of PP-rings and it is related to the compactness of the space of the minimal primes ideals and to the class of weak Baer rings.
\end{abstract}

Keywords: Boolean products, elementary class, continuous sections, Baer rings, PPrings, minimal prime ideales.

Mathematics Subject Classification: 03C20, 16S60, 13J25, 16 G99.

*Centro de Investigación en Matemática Pura y Aplicada (CIMPA) y Escuela de Matemática, Universidad de Costa Rica, Ciudad Universitaria Rodrigo Facio, San José 2060, Costa Rica. E-Mail: jguier@emate.ucr.ac.cr 


\section{Introducción}

Aquí introducimos una noción, inspirados en la proyectabilidad en los $f$-anillos y que llamamos separabilidad en la clase de anillos reducidos. Esta propiedad es evidentemente de primer orden. Por otro lado existe en [7], una noción que resulta evidentemente parecida a la anterior y que determina la clase de PP-anillos. En el artículo de [7], los PP-anillos se estudian en relación con los anillos de funciones continuas a valores reales. En [2], la noción de proyectabilidad se estudia también en relación con los anillos de funciones continuas a valores reales y gracias a estos dos trabajos, se observa que las nociones coinciden en la clase de anillos de funciones continuas a valores reales. La noción de PP-anillo no es a primera vista de primer orden, pues se enuncia con axiomas que involucran proyectabilidad de módulos principales. El autor pensó que la teoría elemental de la clase de PP-anillos podría estar vinculada con la teoría de los anillos separables. Esto resulta ser verdadero, pero en realidad se deduce que la clase de PP-anillos es de primer orden y por tanto, coincide con la clase de anillos separables. Esto es lo que constituye esencialmente la segunda sección de este trabajo.

En la tercera y última sección, se estudia la representación de los anillos separables como anillos de secciones continuas de haces Hausdorff sobre espacios que resultan ser compactos (y booleanos). En [9], el autor menciona bajo la óptica de los PP-anillos, que esta representación es posible. Sin embargo, la demostración que aquí se da de este hecho, nos permite realizar que precisamente la representación se da sólo en el caso en que el espacio de ideales primos minimales sea compacto, agregando una condición "natural" sobre los idempotentes. Esto nos lleva a ligar el concepto de separabilidad con los anillos que son estudiados en [3]. La condición "natural" en los idempotentes es en su esencia, la condición de Baer débil estudiada en [8].

\section{Anillos separables y axiomatización elemental de PP-anillos}

A continuación todos los anillos serán conmutativos y con unidad. En [7] se menciona la noción de PP-anillo, que pide que todo ideal principal sea un módulo proyectivo sobre él mismo.

Definición 2.1 Un anillo $R$ se dice ser un $\mathbf{P P}$-anillo si para todo $a \in R$ se tiene que $(a)$ es un $R$-módulo proyectivo.

Se puede probar el siguiente hecho, que precisa una axiomatización de segundo orden de esta clase de anillos.

Hecho 2.2 Un anillo $R$ es un PP-anillo si y solo si para todo a $\in R$, existe un isomorfismo que hace cierta la aserción siguiente: $(a) \oplus \operatorname{Ann}(a) \cong R$.

Demostración: $(\Leftarrow)$ : Si existe tal isomorfismo entonces $(a)$ es el sumando de un $R$ módulo libre y por tanto ( $a$ ) es un $R$-módulo proyectivo. 
$(\Rightarrow)$ : Consideremos la siguiente sucesión:

$$
0 \longrightarrow \operatorname{Ann}(a) \hookrightarrow R \stackrel{\varphi}{\longrightarrow}(a) \longrightarrow 0,
$$

en donde $\varphi: R \rightarrow(a), r \mapsto r a$ es evidentemente un homomorfismo de $R$-módulos sobreyectivos. Claramente $\operatorname{Ann}(a)=\operatorname{ker}(\varphi)$ y por tanto la sucesión corta es exacta. Como el $R$-módulo $(a)$ es proyectivo, entonces la sucesión se escinde y por tanto $R \cong \operatorname{Ann}(a) \oplus(a)$.

Un elemento teórico importante para estudiar la estructura elemental de la clase de PP-anillos, es la siguiente proposición:

Proposición 2.3 La clase de PP-anillos es cerrada por ultraproductos.

Demostración: Sea $\left(A_{i}\right)_{i \in I}$ una clase de PP-anillos, $\mathcal{D}$ un ultrafiltro sobre el conjunto $I$ y $A=\prod_{i \in I} A_{i} / \mathcal{D}$. Un elemento $\alpha \in A$ es de la forma $\alpha=\left(a_{i}\right)_{i \in I} / \mathcal{D}$. Como cada $A_{i}$ es un PP-anillo, entonces existe un isomorfismo $\varphi_{i}: A_{i} \rightarrow \operatorname{Ann}\left(a_{i}\right) \oplus\left(a_{i}\right)$, para todo $i \in I$. Por tanto

$$
\varphi: \prod_{i \in I} A_{i} \longmapsto\left(\prod_{i \in I} \operatorname{Ann}\left(a_{i}\right)\right) \oplus \prod_{i \in I}\left(a_{i}\right)
$$

dado por $\varphi\left(\left(a_{i}\right)_{i \in I}\right)=\left(\left(\psi_{i}\left(a_{i}\right)\right)_{i \in I},\left(\varphi_{i}\left(a_{i}\right)\right)_{i \in I}\right)$ es un isomorfismo en donde se declara que $\varphi_{i}(a)=\left(\psi_{i}(a), \varphi_{i}(a)\right)$ para todo $a \in A_{i}$ y para todo $i \in I$. Poniendo

$$
\tilde{\varphi}: \prod_{i \in I} A_{i} / \mathcal{D} \longrightarrow\left(\prod_{i \in I} \operatorname{Ann}\left(a_{i}\right) / \mathcal{D}\right) \oplus\left(\prod_{i \in I}\left(a_{i}\right) / \mathcal{D}\right)
$$

dado por medio de

$$
\tilde{\varphi}\left(\left(a_{i}\right)_{i \in I} / \mathcal{D}\right)=\left(\left(\psi_{i}\left(a_{i}\right)\right)_{i \in I} / \mathcal{D},\left(\varphi_{i}\left(a_{i}\right)\right)_{i \in I} / \mathcal{D}\right) .
$$

Se ve claramente que $\tilde{\varphi}$ es un isomorfismo. Ahora observe que se tienen los dos hechos siguientes:

$$
\begin{aligned}
\operatorname{Ann}(\alpha)=\operatorname{Ann}\left(\left(a_{i}\right)_{i \in I} / \mathcal{D}\right) & =\left\{b \in \prod_{i \in I} A_{i} / \mathcal{D}: b \cdot \alpha=0\right\} \\
& =\left\{\left(b_{i}\right)_{i \in I} / \mathcal{D}:\left\{i \in I: b_{i} \alpha_{i}=0\right\} \in \mathcal{D}\right\} \\
& =\left\{\left(b_{i}\right)_{i \in I} / \mathcal{D}:\left\{i \in I: b_{i} \in \operatorname{Ann}\left(\alpha_{i}\right)\right\} \in \mathcal{D}\right\} \\
& =\prod_{i \in I} \operatorname{Ann}\left(\alpha_{i}\right) / \mathcal{D}
\end{aligned}
$$

y

$$
\begin{aligned}
(\alpha) & =\left\{b \in \prod_{i \in I} A_{i} / \mathcal{D}: \text { existe } c \in \prod_{i \in I} A_{i} / \mathcal{D} \text { tal que } b=c \alpha\right\} \\
& =\left\{\left(b_{i}\right)_{i \in I} / \mathcal{D}: \text { existe }\left(c_{i}\right)_{i \in I} \in \prod_{i \in I} A_{i} \text { tal que }\left\{i \in I: b_{i}=c_{i} \alpha_{i}\right\} \in \mathcal{D}\right\} \\
& =\left\{\left(b_{i}\right)_{i \in I} / \mathcal{D}:\left\{i \in I: b_{i} \in\left(\alpha_{i}\right)\right\} \in \mathcal{D}\right\} \\
& =\prod_{i \in I}\left(\alpha_{i}\right) / \mathcal{D} .
\end{aligned}
$$


Entonces por estos dos hechos lo que tenemos es que:

$$
\tilde{\varphi}: \prod_{i \in I} A_{i} / \mathcal{D} \rightarrow \operatorname{Ann}(\alpha) \oplus(\alpha)
$$

es un isomorfismo y por tanto deducimos que $\prod_{i \in I} A_{i} / \mathcal{D}$ es un PP-anillo.

La siguiente proposición es un primer intento de cuestionamiento sobre la naturaleza de la clase de PP-anillos que no resulta nada patológica.

Proposición 2.4 La clase de PP-anillos es cerrada por isomorfismos.

Demostración: Sea $\varphi: A \rightarrow B$ un isomorfismo de anillos y supongamos que $B$ es un PP-anillo. Vamos a ver que $A$ también es un PP-anillo. Sea $a \in A$. Entonces $\varphi(a) \in B$. Existe un isomorfismo $\psi: B \rightarrow(\varphi(a))_{B} \oplus \operatorname{Ann}_{B}(\varphi(a))$.

Entonces $\psi \circ \varphi: A \rightarrow(\varphi(a))_{B} \oplus \operatorname{Ann}_{B}(\varphi(a))$ es un isomorfismo. Está claro que $(\varphi(a))_{B} \subseteq B$ y por medio de $\varphi^{-1}$ se tiene $(\varphi(a))_{B} \rightarrow(a)_{A}, b \cdot \varphi(a) \mapsto \varphi^{-1}(b) \cdot a$ un isomorfismo. También se tiene que $\varphi^{-1}: \operatorname{Ann}_{B}(\varphi(a)) \rightarrow \operatorname{Ann}_{A}(a)$ es un isomorfismo en vista de que $b \cdot \varphi(a)=0$ si y solo si $\varphi^{-1}(b) \cdot a=0$. Entonces $\varphi^{-1} \circ \psi \circ \varphi: A \rightarrow(a)_{A} \oplus \operatorname{Ann}_{A}(a)$ es un isomorfismo; lo que prueba que $A$ es un PP-anillo.

Por resultados conocidos en lógicas de primer orden, para decidir o no si la clase de PP-anillos es una clase elemental, bastaría por medio de la proposición anterior indagar si dicha clase es cerrada por equivalencia elemental. Esto se realizará oportunamente por medio de las subestructuras elementales. La noción que introducimos a continuación, es similar a la de PP-anillos y proviene de los anillos reticulados y en la cual, la polar y bipolar se interpretan (en la clase de anillos reducidos) usando únicamente la estructura de anillo y sin tomar en cuenta la relación de orden parcial existente en los anillos reticulados.

Definición 2.5 Un anillo $A$ es separable si $A=\operatorname{Ann}(a)+\operatorname{Ann}(\operatorname{Ann}(a))$, para todo $a \in A$.

Observe que la noción de separabilidad es de primer orden en el lenguaje de anillos $\mathcal{L}=\{0,1,+, \cdot\}$ pues que un anillo $A$ sea separable equivale a que:

$$
A \models \forall a \forall b(\exists c \exists d(b=c+d \wedge c a=0 \wedge \forall e(a e=0 \rightarrow d e=0))) .
$$

Notemos también que si un anillo $A$ es separable y reducido (i.e.: sin elementos nilpotentes no cero) entonces $A=\operatorname{Ann}(a) \oplus \operatorname{Ann}(\operatorname{Ann}(a))$ para todo $a \in A$ pues si $c \in \operatorname{Ann}(a) \cap$ $\operatorname{Ann}(\operatorname{Ann}(a))$ entonces $c^{2}=0$ y por tanto $c=0$.

También se tiene que $(a) \subseteq \operatorname{Ann}(\operatorname{Ann}(a))$ pues si $\gamma \in(a)$ entonces $\gamma=c a$ para algún $c \in A$ y $\gamma \in \operatorname{Ann}(\operatorname{Ann}(a))$ quiere decir que $\gamma d=0$ para todo $d \in \operatorname{Ann}(a)$, lo que es evidente en vista de la forma que tiene $\gamma$. Ahora, no necesariamente $(a)=\operatorname{Ann}(\operatorname{Ann}(a))$ pues si $A=\mathbb{Z}$ y tomando $(2) \subsetneq \operatorname{Ann}(\operatorname{Ann}(2))=\operatorname{Ann}(\{0\})=\mathbb{Z}$. En realidad este contraejemplo es cierto independientemente del anillo íntegro que se tome. Si hubiera sido cierto que $(a)=\operatorname{Ann}(\operatorname{Ann}(a))$, entonces todo anillo separable sería un PP-anillo. No obstante se tiene: 
Proposición 2.6 Sea A un anillo conmutativo con unidad y reducido. Si A es separable entonces $A$ es un PP-anillo.

Demostración: Sea $a \in A$. Lo que tenemos es que $A=\operatorname{Ann}(a) \oplus \operatorname{Ann}(\operatorname{Ann}(a))$. Como $1 \in A$ entonces $1=c(a)+d(a)$ con $c(a) \in \operatorname{Ann}(a)$ y $d(a) \in \operatorname{Ann}(\operatorname{Ann}(a))$. Claramente se tiene que $(d(a)) \subseteq \operatorname{Ann}(\operatorname{Ann}(a))$. Por otro lado si $b \in \operatorname{Ann}(\operatorname{Ann}(a))$ entonces $b=b \cdot 1=$ $b \cdot(c(a)+d(a))=b \cdot c(a)+b \cdot d(a)=0+b \cdot d(a)=b \cdot d(a)$ y por tanto $b \in(d(a))$. Esto nos muestra que $\operatorname{Ann}(\operatorname{Ann}(a))=(d(a))$.

Consideremos ahora $\varphi:(d(a)) \rightarrow(a), b \cdot d(a) \mapsto b \cdot a$. Debemos ver que $\varphi$ está bien definido, es decir; si $b_{1} \cdot d(a)=b_{2} \cdot d(a)$ para algunos $b_{1}, b_{2} \in A$ entonces $b_{1} \cdot d(a) \cdot a=b_{2} \cdot d(a) \cdot a$ y luego $b_{1} \cdot a=b_{2} \cdot a$ pues $a=a \cdot 1=a \cdot(c(a)+d(a))=a \cdot c(a)+a \cdot d(a)=0+a \cdot d(a)=a \cdot d(a)$. Consecuentemente $\varphi$ está bien definido. Evidentemente $\varphi$ perserva la estructura de $A$ módulo de $(d(a))$ y de $(a)$. Claramente $\varphi$ es sobreyectiva. Veamos ahora que $\varphi$ es inyectiva: sea $\gamma \in(d(a))$ tal que $\varphi(\gamma)=0$. Se tiene que $\gamma=b \cdot d(a)$ con $b \in A$ y $b \cdot a=0$. Esto nos dice que $b \in \operatorname{Ann}(a)$ y como $d(a) \in \operatorname{Ann}(\operatorname{Ann}(a))$ entonces $b \cdot d(a)=0$, es decir $\gamma=0$.

Esto nos muestra que $\varphi$ es biyectiva y por tanto existe un isomorfismo de $A$-módulos que hace $\operatorname{Ann}(\operatorname{Ann}(a)) \cong(a)$. Por tanto $A=\operatorname{Ann}(a) \oplus \operatorname{Ann}(\operatorname{Ann}(a)) \cong \operatorname{Ann}(a) \oplus(a)$ y esto quiere decir precisamente que $A$ es un PP-anillo.

En vista de indagar más sobre la elementaridad de la clase de PP-anillos, mejoramos la presentación del hecho 2.2 a la siguiente proposición:

Proposición 2.7 Sea A un anillo cualquiera. Entonces son equivalentes:

(i) A es un PP-anillo,

(ii) para todo $a \in A$, existe $\varphi$ un isomorfismo de $A$-módulos tal que $A \cong(a) \oplus \operatorname{Ann}(a)$,

(iii) para todo $a \in A$, se tiene que $A=C \oplus \operatorname{Ann}(a)$ y existe $\psi$ un isomorfismo de $A$ módulos tal que $\psi:(a) \rightarrow C$.

Demostración: (i) $\Rightarrow$ (iii): Sea $a \in A$. Se tiene que $(a)$ es un $A$-módulo proyectivo. Consideremos la siguiente sucesión $0 \rightarrow \operatorname{Ann}(a) \hookrightarrow A \rightarrow(a) \rightarrow 0$ en donde $\varphi: A \rightarrow$ $(a), r \mapsto r a$. Claramente $\varphi$ es un homomorfismo de $A$-módulos que es sobreyectivo. Evidentemente $\operatorname{ker}(\varphi)=\operatorname{Ann}(a)=\operatorname{im}(i)$. Entonces dicha sucesión es exacta y por ser (a) un $A$-módulo proyectivo, dicha sucesión se escinde. Sea $s$ una sección de $\varphi$, es decir $s:(a) \rightarrow A$ un homomorfismo de $A$-módulos tal que $\varphi \circ s=\operatorname{id}_{(a)}$. Tomando $C=\operatorname{im}(s)$ se sabe que $A=\operatorname{Ann}(a) \oplus C$ y claramente $s$ es inyectiva y por tanto $C$ es isomorfo a $(a)$.

(iii) $\Rightarrow$ (ii): Es evidente pues basta tomar $\varphi$ el isomorfismo dado por $\psi \oplus \operatorname{id}_{\operatorname{Ann}(a)}$.

(ii) $\Rightarrow$ (i): Ya fue mostrado anteriormente.

Proposición 2.8 Si $B$ es un PP-anillo y $A \prec B$ entonces $A$ es un PP-anillo. 
Demostración: Sea $A$ un anillo cualquiera y sea $B$ un PP-anillo tal que $A \prec B$. Queremos ver si $A$ es también un PP-anillo. Sea $a \in A$ y entonces considerando $a \in B$ se tiene que $B=C \oplus \operatorname{Ann}_{B}(a)$ y que existe $\psi$ un isomorfismo de $B$-módulos tal que $\psi:(a)_{B} \rightarrow C$. Ahora se tiene que $C=\operatorname{im}(\psi)=\{\psi(r a): r \in B\}=\{r \cdot \psi(a): r \in B\}=(\psi(a))_{B}$. Entonces $B=(\psi(a))_{B} \oplus \operatorname{Ann}_{B}(a)$.

Veamos ahora que $\operatorname{Ann}_{B}(\psi(a))=\operatorname{Ann}_{B}(a)$. Sea $r \in B$ tal que $r \in \operatorname{Ann}_{B}(\psi(a))$, entonces $r \cdot \psi(a)=0$ y esto implica que $\psi(r a)=0$. Como $\psi$ es inyectivo entonces $r a=0 \mathrm{y}$ por tanto $r \in \operatorname{Ann}_{B}(a)$. Entonces se tiene que $\operatorname{Ann}_{B}(\psi(a)) \subseteq \operatorname{Ann}_{B}(a)$. Ahora sea $r \in B$ tal que $r \in \operatorname{Ann}_{B}(a)$, es decir $r a=0$. Al aplicar $\psi$ se obtiene que $\psi(r a)=\psi(0)=0$ y por tanto $r \cdot \psi(a)=0$; es decir $r \in \operatorname{Ann}_{B}(\psi(a))$. Entonces $\operatorname{Ann}_{B}(a) \subseteq \operatorname{Ann}_{B}(\psi(a))$ y por tanto la igualdad.

Entonces lo que se tiene es que $B=(\psi(a))_{B} \oplus \operatorname{Ann}_{B}(a)$ y $\operatorname{Ann}_{B}(\psi(a))=\operatorname{Ann}_{B}(a)$. Esto se puede decir mediante una fórmula de primer orden que es la siguiente:

$$
B \models \forall x \exists ! y \exists ! z\left(x=y+z \wedge y \in(\psi(a)) \wedge z-a=0 \wedge \operatorname{Ann}_{B}(\psi(a))=\operatorname{Ann}_{B}(a)\right),
$$

o lo que es lo mismo:

$$
B \models \forall x \exists ! y \exists ! z(x=y+z \wedge \exists w(y=w \cdot \psi(a)) \wedge z \cdot a=0 \wedge \forall t(t \cdot \psi(a)=0 \leftrightarrow t \cdot a=0)) .
$$

En realidad esto prueba que:

$$
B \models \exists \delta \forall x \exists ! y \exists ! z(x=y+z \wedge \exists w(y=w \cdot \delta) \wedge z \cdot a=0 \wedge \forall t(t \cdot \delta=0 \leftrightarrow t \cdot a=0)) .
$$

Ahora ya solo tenemos parámetros en $A$ y como $A \prec B$ entonce se tiene que:

$$
A \models \exists \delta \forall x \exists ! y \exists ! z(x=y+z \wedge \exists w(y=w \cdot \delta) \wedge z \cdot a=0 \wedge \forall t(t \cdot \delta=0 \leftrightarrow t \cdot a=0)) .
$$

Tomemos $\delta \in A$ tal que:

$$
A \models \forall x \exists ! y \exists ! z(x=y+z \wedge \exists w(y=w \cdot \delta) \wedge z \cdot a=0 \wedge \forall t(t \cdot \delta=0 \leftrightarrow t \cdot a=0)) .
$$

Esto lo que quiere decir es que $A=(\delta)_{A} \oplus \operatorname{Ann}_{A}(a)$ y $\operatorname{Ann}_{A}(\delta)=\operatorname{Ann}_{A}(a)$. Ahora se define $\rho:(\delta)_{A} \rightarrow(a)_{A}$ mediante $\rho(r \delta)=r a$ para todo $r \in A$. Primero veamos que $\rho$ está bien definido pues podría ser que $r_{1} \delta=r_{2} \delta$ para algunos $r_{1}, r_{2} \in A$. Pero en este caso $\left(r_{1}-r_{2}\right) \delta=0$ y por tanto $r_{1}-r_{2} \in \operatorname{Ann}(\delta)=\operatorname{Ann}(a)$; es decir $r_{1} a=r_{2} a$. Se prueba similarmente que $\rho$ es inyectivo. Claramente $\rho$ es sobreyectivo y un homomorfismo de $A$-módulos. Entonces $\rho$ es un isomorfismo de $A$-módulos.

Por tanto $A=C \oplus \operatorname{Ann}_{A}(a)$ con $\rho: C \rightarrow(a)_{A}$ un isomorfismo de $A$-módulos. Como esto es válido para todo $a \in A$ se tiene que $A$ es un PP-anillo.

Es conocido que una clase de estructuras es elemental si y solo si dicha clase es cerrada por ultraproductos y por equivalencia elemental. Debido al resultado de Keisler-Shelah que caracteriza la equivalencia elemental de dos estructuras en relación a un isomorfismo de ultrapotencias de las estructuras originales, se tiene que la proposición anterior junto con la proposición 2.3, conlleva al resultado siguiente: 
Proposición 2.9 La clase de PP-anillos es elemental en el lenguaje de anillos.

De hecho la demostración de la proposición 2.8 contiene una fórmula de primer orden que axiomatiza la clase de PP-anillos y que está dada por

$$
\forall a \exists \delta \forall x \exists ! y \exists ! z(x=y+z \wedge \exists w(y=w \cdot \delta) \wedge z \cdot a=0 \wedge \forall t(t \cdot \delta=0 \leftrightarrow t \cdot a=0)) .
$$

Es importante mencionar el hecho evidente de que para $M$ un $R$-módulo y para $M_{1}$ y $M_{2}$ dos $R$-submódulos definibles en el lenguaje de anillos por medio de fórmulas $\varphi_{1}(x)$ y $\varphi_{2}(x)$, se tiene que $M=M_{1} \oplus M_{2}$ si y solo si $M \models \forall x \exists ! y \exists ! z\left(x=y+z \wedge \varphi_{1}(y) \wedge \varphi_{2}(z)\right)$. Basta con recordar que $M=M_{1} \oplus M_{2}$ equivale a que $M=M_{1}+M_{2}$ y $M_{1} \cap M_{2}=\{0\}$. Entonces lo anteriormente mencionado prueba que:

Proposición 2.10 Sea $A$ un anillo cualquiera. Entonces $A$ es un PP-anillo si y solo si

$$
A \models \forall a \exists \delta \forall x \exists ! y \exists ! z(x=y+z \wedge \exists w(y=w \cdot \delta) \wedge z \cdot a=0 \wedge \forall t(t \cdot \delta=0 \leftrightarrow t \cdot a=0)) .
$$

El hecho de poder exhibir y comprender mejor la estructura de los PP-anillos permite probar el siguiente hecho:

Proposición 2.11 Si A es un PP-anillo con unidad entonces A es separable.

Demostración: Como $A$ es un PP-anillo entonces $A$ satisface la fórmula de la proposición 2.10. Entonces si $a \in A$ es cualquier elemento, sea $\delta \in A$ tal que $A=(\delta) \oplus \operatorname{Ann}(a)$ y $\operatorname{Ann}(a)=\operatorname{Ann}(\delta)$. Ahora escribamos $1 \in A$ como $1=c(a)+d(a)$ con $c(a) \in \operatorname{Ann}(a)$ y $d(a) \in(\delta)$. Entonces $\delta \mid d(a)$ y por tanto $(d(a)) \subseteq(\delta)$.

Veamos que $\operatorname{Ann}(\operatorname{Ann}(a)) \subseteq(d(a))$. Sea $x \in A$ con $x \in \operatorname{Ann}(\operatorname{Ann}(a))$, es decir $x \cdot l=0$ para todo $l \in A$ con $l \cdot a=0$. Como $c(a) \in \operatorname{Ann}(a)$ entonces $x \cdot c(a)=0$ y por tanto $x=x \cdot 1=x \cdot(c(a)+d(a))=x \cdot c(a)+x \cdot d(a)=0+x \cdot d(a)=x \cdot d(a)$. Lo que esto está probando es que $x \in(d(a))$. Esto nos permite obtener que $\operatorname{Ann}(\operatorname{Ann}(a)) \subseteq(d(a)) \subseteq(\delta)$.

Ahora vamos a demostrar que $(\delta) \subseteq \operatorname{Ann}(\operatorname{Ann}(a))$. Sea $x \in(\delta)$, es decir $x=r \cdot \delta$ con $r \in A$. Queremos ver que $x \in \operatorname{Ann}(\operatorname{Ann}(a))$, entonces consideremos $l \in A$ tal que $l \in \operatorname{Ann}(a)$, es decir $l \in \operatorname{Ann}(\delta)$ y por tanto $l \cdot \delta=0$. Ahora $x \cdot l=(r \delta) \cdot l=r \cdot(\delta l)=$ $r \cdot(l \delta)=r \cdot 0=0$. Esto lo que está probando es que $x \in \operatorname{Ann}(l)$ para todo $l \in \operatorname{Ann}(a)$; es decir $x \in \operatorname{Ann}(\operatorname{Ann}(a))$.

Con esta última afirmación se tiene que $(\delta)=(d(a))=\operatorname{Ann}(\operatorname{Ann}(a))$ lo que a su vez permite concluir que $A=\operatorname{Ann}(a) \oplus \operatorname{Ann}(\operatorname{Ann}(a))$ para todo $a \in A$.

Podemos resumir las proposiciones 2.6 y 2.11 mediante el teorema siguiente:

Teorema 2.12 Si $A$ es un anillo conmutativo, reducido y con unidad entonces $A$ es separable si y solo si $A$ es un PP-anillo.

Observemos que este teorema da otra caracterización elemental de la clase de PPanillos que no era del todo obvia. 


\section{Anillos separables y anillos de Baer}

En esta sección, se demuestra que los anillos separables o PP-anillos se representan por medio de secciones continuas de haces Haussdorff de anillos íntegros. Este resultado en sí no es original pues se menciona en [9], pero la demostración que aquí se presenta, permite relacionar estos anillos con la compacidad del espacio de ideales primos minimales (anillos de Henriksen-Jerison) y con los anillos de Baer y de Baer débil.

La representación se efectúa sobre el espacio de ideales primos minimales que ha sido ampliamente estudiado. Tomamos como referencia el artículo [3]. Denotamos por $\mathfrak{m}(A)$ el conjunto de ideales primos minimales de un anillo $A$. A continuación vamos a detallar varios resultados de [3] que necesitamos en el desarrollo de la presente sección. Se sabe que $\bigcap\{p: p \in \mathfrak{m}(A)\}=\bigcap_{p \in \mathfrak{m}(A)} p=\eta(A)$ el conjunto de elementos nilpotentes de $A$. También es cierto que un ideal primo $p$ de $A$ es minimal si y solo si para todo $x \in p$, existe $a \in A \backslash p$ tal que $a x$ es nilpotente. En particular si $A$ es un anillo reducido, un ideal primo $p$ de $A$ es minimal si y solo si para todo $x \in p$, existe $a \in A \backslash p$ tal que $a x=0$. Esto conlleva a que si $p$ es un ideal primo minimal entonces $p=\bigcup_{a \in A \backslash p} \operatorname{Ann}(a)$.

Para $S \subseteq A$, se toma $V(S)=\{p \in \mathfrak{m}(A): S \subseteq p\}$. El conjunto de los $V(a)=$ $\{p \in \mathfrak{m}(A): a \in p\}$ para $a \in A$ es una base de cerrados para una topología de $\mathfrak{m}(A)$. Para $S \subseteq A$ si se toma $\operatorname{Ann}(S)=\{a \in A: a \cdot S=0\}$, en [3] lográn probar en el caso de un anillo reducido que $V(\operatorname{Ann}(a))=\mathfrak{m}(A) \backslash V(a)$ para todo $a \in A$. Denotamos por $D(a)=\mathfrak{m}(A) \backslash V(a)=\{p \in \mathfrak{m}(A): a / \notin\}$ para todo $a \in A$. Entonces lo que han probado es que $\{D(a): a \in A\}$ es una base de abiertos-cerrados para los abiertos de $\mathfrak{m}(A)$. Entonces $\mathfrak{m}(A)$ es un espacio Haussdorff con una base de abiertos-cerrados. Observe que esta topología de $\mathfrak{m}(A)$ es la topología heredada de la topología de Zariski de $\operatorname{Spec}(A)=\{p: p$ es un ideal primo de $A\}$.

En [3] se dice que un anillo $A$ satisface la condición del anulador si para todo $x, y \in A$ existe $z \in A$ tal que $\operatorname{Ann}(x) \cap \operatorname{Ann}(y)=\operatorname{Ann}(z)$. Ahí mismo se demuestra que esta condición es equivalente a que para todo $x, y \in A$, existe $z \in A$ tal que $V(x) \cap V(y)=V(z)$. Siempre se tiene que $V(x) \cup V(y)=V(x y)$. Los autores en [3] demuestran que para un anillo reducido $A$, que el espacio $\mathfrak{m}(A)$ sea compacto y que $A$ satisfaga la condición del anulador, equivale a que el espacio $\mathfrak{m}(A)$ es compacto y $\{V(a): a \in A\}$ es una base para los abiertos de $\mathfrak{m}(A)$ y esto equivale también a que para todo $x \in A$, existe $x^{\prime} \in A$ tal que $\operatorname{Ann}(x)=\operatorname{Ann}\left(\operatorname{Ann}\left(x^{\prime}\right)\right)$. Si un anillo $A$ satisface alguna de las tres condiciones equivalentes anteriores, nosotros diremos que el anillo $A$ satisface la condición de Henriksen-Jerison o que es de Henriksen-Jerison.

La siguiente proposición liga la sección anterior con lo que previamente discutimos.

Proposición 3.1 Sea $A$ un anillo conmutativo, reducido y con unidad. Si A es separable entonces $A$ es de Henriksen-Jerison.

Demostración: Sea $a \in A$. Se sabe que $1=c(a)+d(a)$ con $c(a) \in \operatorname{Ann}(a)$ y $d(a) \in$ $\operatorname{Ann}(\operatorname{Ann}(a))$. Bastaría probar que $\operatorname{Ann}(\operatorname{Ann}(c(a)))=\operatorname{Ann}(a)$. 
Sea $\alpha \in \operatorname{Ann}(\operatorname{Ann}(c(a)))$; esto quiere decir que $\alpha y=0$ para todo $y \in \operatorname{Ann}(c(a))$. Como $c(a) \in \operatorname{Ann}(a)$ entonces $a \in \operatorname{Ann}(c(a))$ y esto permite concluir que $\alpha a=0$. Entonces se tiene la inclusión $\operatorname{Ann}(\operatorname{Ann}(c(a))) \subseteq \operatorname{Ann}(a)$.

Sea ahora $\alpha \in \operatorname{Ann}(a)$. Tomemos $y \in \operatorname{Ann}(c(a))$ arbitrario, entonces $y \cdot c(a)=0$ lo que permite concluir que $y=y \cdot 1=y \cdot(c(a)+d(a))=y \cdot c(a)+y \cdot d(a)=0+y \cdot d(a)=$ $y \cdot d(a)$. Entonces $y \in(d(a))$ y como $d(a) \in \operatorname{Ann}(\operatorname{Ann}(c(a)))$ entonces se tiene que $(d(a)) \subseteq \operatorname{Ann}(\operatorname{Ann}(c(a)))$. Consecuentemente $y \in \operatorname{Ann}(\operatorname{Ann}(c(a)))$. Como $\alpha \in \operatorname{Ann}(a)$ entonces $y \cdot \alpha=0$. Esto quiere decir que $\alpha \cdot y=0$ para todo $y \in \operatorname{Ann}(c(a))$; es decir $\alpha \in \operatorname{Ann}(\operatorname{Ann}(c(a)))$. Hemos probado la inclusión $\operatorname{Ann}(a) \subseteq \operatorname{Ann}(\operatorname{Ann}(c(a)))$ y con esto la igualdad.

Como corolario de esta proposición 3.1 y del teorema anteriormente mencionado de [3], se tiene que:

Proposición 3.2 Sea $A$ un anillo reducido y con unidad. Si A es separable entonces $\mathfrak{m}(A)$ es compacto.

La siguiente proposición guarda en sí una condición interesante.

Proposición 3.3 Sea A un anillo reducido y con unidad. Si A es separable entonces para todo $a \in A$ existe un idempotente $e \in A$ tal que $V(a)=V(e)$ y $D(a)=V(1-e)$.

Demostración: Para $a \in A$, existe $c(a) \in \operatorname{Ann}(a)$ y $d(a) \in \operatorname{Ann}(\operatorname{Ann}(a))$ tal que $1=$ $c(a)+d(a)$. Se tiene que $c(a) \cdot d(a)=0$ y entonces $c(a)=c(a) \cdot 1=c(a) \cdot(c(a)+d(a))=$ $c(a)^{2}+0=c(a)^{2}$ y también $d(a)=d(a) \cdot 1=d(a) \cdot(c(a)+d(a))=0+d(a)^{2}=d(a)^{2}$. Entonces $c(a)$ y $d(a)$ son idempotentes de $A$.

Ahora probemos que $V(a)=V(d(a))$. Primero que todo, si $p \in \mathfrak{m}(A)$ es tal que $d(a) \in p$ entonces $a=a \cdot d(a) \in p$. Ahora si $p \in \mathfrak{m}(A)$ es tal que $a \in p$, como $p$ es minimal existe $x \in A \backslash p$ tal que $a x=0$. Esto quiere decir que $x \in \operatorname{Ann}(a)$ y como $d(a) \in \operatorname{Ann}(\operatorname{Ann}(a))$ entonces $x \cdot d(a)=0 \in p$. Como $x \notin p$ entonces $d(a) \in p$. Esto prueba entonces que $V(a)=V(d(a))$.

Evidentemente $D(a)=D(d(a))$. Sea $p \in \mathfrak{m}(A)$ tal que $a \notin p$. Entonces $d(a) \notin p$ y como $c(a) \cdot d(a)=0 \in p$ se tiene forzosamente que $c(a) \in p$. Ahora sea $p \in \mathfrak{m}(A)$ tal que $c(a) \in p$. Entonces $d(a) \notin p$ pues de lo contrario $c(a)+d(a)=1 \in p$ que es una contradicción y por tanto $a \notin p$. Esto prueba entonces que $D(a)=V(c(a))=V(1-d(a))$.

Las proposiciones 3.1 y 3.3 las resumimos en lo siguiente:

Proposición 3.4 Sea $A$ un anillo reducido y con unidad. Si $A$ es separable entonces $\mathfrak{m}(A)$ es compacto y para todo $a \in A$ existe un idempotente $e \in A$ tal que $V(a)=V(e)$ y $D(a)=V(1-e)$. 
Para un anillo $A$, sea $\mathbf{X}(A)=\biguplus_{p \in \mathfrak{m}(A)} A / p$ el espacio estrellado de $A$. Vamos a dotar a $\mathbf{X}(A)$ de una topología y con este fin, introducimos la siguiente notación. Para cada $a \in A$, consideremos $\hat{a}: \mathfrak{m}(A) \rightarrow \mathbf{X}(A)$ dada por $\hat{a}(p)=a+p$ para todo $p \in \mathfrak{m}(A)$. Ahora tomemos en $\mathbf{X}(A)$ la topología más grande (o más débil) que hace todos los elementos de $\{\hat{a}: a \in A\}$ continuos. Es decir, sea $\mathcal{U}$ la topología de $\mathbf{X}(A)$ definida por medio de:

$$
\mathcal{U}=\left\{\mathcal{O} \subseteq \mathbf{X}(A): \hat{a}^{-1}(\mathcal{O}) \text { es abierto en } \mathfrak{m}(A), \text { para todo } a \in A\right\}
$$

Ahora consideremos $\Gamma(\mathfrak{m}(A), \mathbf{X}(A))$ el conjunto de todas las secciones continuas de $\mathfrak{m}(A)$ en $\mathbf{X}(A)$. Es decir:

$$
\Gamma(\mathfrak{m}(A), \mathbf{X}(A))=\{s: \mathfrak{m}(A) \rightarrow \mathbf{X}(A): s \text { es una sección continua }\} .
$$

Recordemos que $s: \mathfrak{m}(A) \rightarrow \mathbf{X}(A)$ es una sección si existe $t: \mathbf{X}(A) \rightarrow \mathfrak{m}(A)$ una función tal que $t \circ s=\operatorname{id}_{\mathbf{X}(A)}$, o equivalentemente si $s(p) \in A / p$ para todo $p \in \mathfrak{m}(A)$.

Se nos hace necesario probar algunos resultados que enunciamos a continuación:

Lema 3.5 Para cualquier anillo $A$, si $s \in \Gamma(\mathfrak{m}(A), \mathbf{X}(A))$ entonces

$$
\llbracket s=\hat{0} \rrbracket=\{p \in \mathfrak{m}(A): s(p)=0+p\}
$$

es un abierto y cerrado de $\mathfrak{m}(A)$.

Demostración: Como $s$ y $\hat{0}$ son continuas, entonces claramente $\llbracket s=\hat{0} \rrbracket$ es un cerrado de $\mathfrak{m}(A)$. Denotemos ahora por $\mathcal{O}_{0}=\{0+p \in \mathbf{X}(A): p \in \mathfrak{m}(A)\}$ y mostremos que $\mathcal{O}_{0}$ es un abierto de $\mathbf{X}(A)$. Para esto nos hace falta ver:

$$
\begin{aligned}
\hat{a}^{-1}\left(\mathcal{O}_{0}\right) & =\left\{p \in \mathfrak{m}(A): \hat{a}(p) \in \mathcal{O}_{0}\right\} \\
& =\{p \in \mathfrak{m}(A): a+p=0+p\} \\
& =\{p \in \mathfrak{m}(A): a \in p\} \\
& =V(a)
\end{aligned}
$$

que es un abierto, para todo $a \in A$. Se tiene entonces que $\mathcal{O}_{0}$ es un abierto de $\mathbf{X}(A)$ y como $s$ es continua entonces

$$
s^{-1}\left(\mathcal{O}_{0}\right)=\left\{p \in \mathfrak{m}(A): s(p) \in \mathcal{O}_{0}\right\}=\{p \in \mathfrak{m}(A): s(p)=0+p\}=\llbracket s=\hat{0} \rrbracket
$$

es un abierto de $\mathfrak{m}(A)$.

Lema 3.6 Sea $A$ un anillo reducido y con unidad. Si A es de Henriksen-Jerison entonces todo abierto-cerrado de $\mathfrak{m}(A)$ es de la forma $V(a)$ para algún a $\in A$.

Demostración: Por hipótesis se tiene que $\mathfrak{m}(A)$ es compacto y que $\{V(a): a \in A\}$ es una base para los abiertos de $\mathfrak{m}(A)$. Sea $\mathcal{O}$ un abierto-cerrado de $\mathfrak{m}(A)$ entonces $\mathcal{O}=\bigcup_{i \in I} V\left(a_{i}\right)$ para algunos $a_{i} \in A$. Como $\mathcal{O}$ es cerrado dentro de un compacto entonces es compacto y 
se tiene $I^{\prime} \subseteq I$ finito tal que $\mathcal{O}=\bigcup_{i \in I^{\prime}} V\left(a_{i}\right)$. Por tanto $\mathcal{O}=V\left(a_{1}\right) \cup \cdots V\left(a_{n}\right)=V(\alpha)$ en donde $\alpha=a_{1} \cdots a_{n} \in A$.

La siguiente proposición se enuncia de la manera más general posible para luego ir agregando hipótesis y lograr el isomorfismo deseado.

Proposición 3.7 Si $A$ es un anillo reducido entonces

$$
\begin{aligned}
\varphi: A & \longrightarrow \Gamma(\mathfrak{m}(A), \mathbf{X}(A)) \\
a & \longmapsto \hat{a},
\end{aligned}
$$

es un homomorfismo de anillos inyectivo.

Demostración: Es un simple cálculo ver que $\varphi$ es un homomorfismo de anillos. Veamos únicamente que $\varphi$ es inyectivo. Sea $a \in A$ tal que $\varphi(a)=\hat{a}=\hat{0}$. Lo que esto quiere decir es que $\hat{a}(p)=\hat{0}(p)$ para todo $p \in \mathfrak{m}(A)$, y por tanto $a+p=0+p$ para todo $p \in \mathfrak{m}(A)$. Consecuentemente $a \in p$ para todo $p \in \mathfrak{m}(A)$; es decir $a \in \bigcap_{p \in \mathfrak{m}(A)} p=\eta(A)=\{0\}$ pues el anillo $A$ es reducido. Entonces $a=0$ y esto prueba la inyectividad.

Proposición 3.8 Sea $A$ un anillo reducido y con unidad. Si A es un anillo de HenriksenJerison y si para todo $a \in A$ existe un idempotente $e \in A$ tal que $V(a)=V(e)$ entonces

$$
\begin{aligned}
\varphi: A & \longrightarrow \Gamma(\mathfrak{m}(A), \mathbf{X}(A)) \\
a & \longmapsto \hat{a},
\end{aligned}
$$

es un isomorfismo.

Demostración: Por la proposición 3.7 lo único que falta probar es que $\varphi$ es sobreyectivo. Sea entonces $s \in \Gamma(\mathfrak{m}(A), \mathbf{X}(A))$. Claramente se tiene que $\mathfrak{m}(A)=\bigcup_{a \in A} \llbracket s=\hat{a} \rrbracket$. Se tiene que $\llbracket s=\hat{a} \rrbracket$ es un abierto-cerrado de $\mathfrak{m}(A)$ para todo $a \in A$ pues $\llbracket s=\hat{a} \rrbracket=\llbracket s-\hat{a}=\hat{0} \rrbracket$ y $s-\hat{a}$ es continua, para todo $a \in A$. Por compacidad de $\mathfrak{m}(A)$ existen $a_{1}, \ldots, a_{n} \in A$ tales que $\bigcup_{i=1}^{n} \llbracket s=\hat{a}_{i} \rrbracket$. Podemos suponer sin pérdida de generalidad que los conjuntos $\left\{\llbracket s=\hat{a_{i}} \rrbracket: i=1, \ldots, n\right\}$ son disjuntos dos a dos. Si denotamos por $\mathcal{O}_{i}=\llbracket s=\hat{a_{i}} \rrbracket$, lo que se tiene es que $s=a_{1 \digamma_{\mathcal{O}_{1}}} \cup a_{2 \digamma_{\mathcal{O}_{2}}} \cup \cdots \cup a_{n \Upsilon_{\mathcal{O}_{n}}}$. Es decir:

$$
s(x)=\left\{\begin{array}{ccc}
a_{1}(x) & \text { si } & x \in \mathcal{O}_{1}, \\
a_{2}(x) & \text { si } & x \in \mathcal{O}_{2}, \\
\vdots & & \vdots \\
a_{n}(x) & \text { si } & x \in \mathcal{O}_{n} .
\end{array}\right.
$$

Observe que si los conjuntos $\left\{\mathcal{O}_{i}: i=1, \ldots, n\right\}$ no hubiesen sido disjuntos dos a dos, entonces el hecho de hacerlos disjuntos dos a dos, corresponde a hacer una escogencia del nombre que se le da a $s$ en la intersección de los $\mathcal{O}$ 's. 
Ahora por el lema 3.6, para cada $i=1, \ldots, n$ existe $b_{i} \in A$ tal que $\mathcal{O}_{i}=V\left(b_{i}\right)$. Además por la hipótesis adicional sobre el anillo $A$, para cada $i=1, \ldots, n$ existe $e_{i} \in A$ un idempotente tal que $\mathcal{O}_{i}=V\left(e_{i}\right)$. Tomando $f_{i}=1-e_{i}$ el idempotente opuesto a $e_{i}$, se tiene que $\mathcal{O}_{i}=D\left(f_{i}\right)$ para todo $i=1, \ldots, n$. Ahora tomemos el elemento $a=\sum_{i=1}^{n} a_{i} f_{i}=$ $a_{1} f_{1}+a_{2} f_{2}+\cdots+a_{n} f_{n} \in A$ y mostremos que $s=\hat{a}$.

Sea $p \in \mathfrak{m}(A)$ cualquiera. Por tanto existe $i \in\{1, \ldots, n\}$ tal que $p \in \llbracket s=\hat{a_{i}} \rrbracket=\mathcal{O}_{i}=$ $V\left(b_{i}\right)=V\left(e_{i}\right)=D\left(f_{i}\right)$ y $p \notin \llbracket s=\hat{a_{j}} \rrbracket=\mathcal{O}_{j}=V\left(b_{j}\right)=V\left(e_{j}\right)=D\left(f_{j}\right)$ para todo $j \neq i$. Esto quiere decir que $f_{i} \notin p$ y $f_{j} \in p$ para todo $j \neq i$. Entonces $f_{i}+p \neq 0$ y $f_{j}+p=0$ para todo $j \neq i$. Como $f_{i}+p$ es un idempotente no cero en $A / p$ un anillo íntegro entonces $f_{i}+p=1+p$. Entonces:

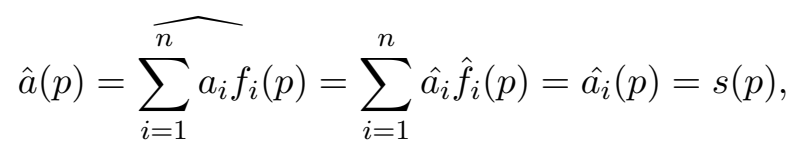

y esto para cualquier $p \in \mathfrak{m}(A)$. Queda entonces demostrado que $s=\hat{a}$ y la sobreyectividad de $\varphi$.

Como corolario tenemos lo siguiente:

Corolario 3.9 Sea $A$ un anillo reducido y con unidad. Si A es separable entonces:

$$
\begin{aligned}
\varphi: A & \longrightarrow \Gamma(\mathfrak{m}(A), \mathbf{X}(A)) \\
a & \longmapsto \hat{a},
\end{aligned}
$$

es un isomorfismo de anillos.

Demostración: Basta usar las proposiciones 3.1, 3.3 y 3.8 .

Este corolario puede decirse usando la terminología de productos booleanos de la siguiente manera: si $A$ es un anillo reducido y con unidad que además es separable entonces $A$ es un producto booleano de anillos íntegros. Ahora, si se tuviera $A$ un producto booleano de anillos íntegros (es decir, un anillo $A$ que sea isomorfo a las secciones continuas de un espacio booleano en un espacio estrellado de anillos íntegros) entonces se puede probar fácilmente que $A$ es separable pues para $a, b \in A$, se tiene que $a a=0 \rrbracket$ y $a \neq 0 \rrbracket$ son abiertos cerrados del espacio booleano y tomando $c=b_{\uparrow_{[a=0]}} \cup 0_{\uparrow_{[a \neq 0]}}$ y $d=0_{\uparrow_{[a=0]}} \cup b_{\uparrow_{[a \neq 0]}}$ uno se da cuenta que $c \in \operatorname{Ann}(a)$ y $d \in \operatorname{Ann}(\operatorname{Ann}(a))$ además de que $b=c+d$. Entonces esto permite ver a grosso modo, que los anillos separables son los únicos que se representan como secciones continuas de haces Hausdorff de anillos íntegros sobre espacios booleanos.

La proposición siguiente relaciona de otra manera los anillos separables y los de Henriksen-Jerison, agregando algunas condiciones sobre los idempotentes. Luego veremos que estas condiciones sobre los idempotentes son conocidas.

Proposición 3.10 Sea A un anillo reducido y con unidad. Las siguientes afirmaciones son equivalentes:

(i) A es separable, 
(ii) A es de Henriksen-Jerison y para todo a $\in A$ existe $e \in A$ un idempotente tal que $V(a)=V(e)$

(iii) $\mathfrak{m}(A)$ es compacto y para todo a $\in A$ existe $e \in A$ un idempotente tal que $V(a)=$ $V(e)$.

Demostración: (i) $\Rightarrow$ (iii): Se tiene gracias a la proposición 3.4.

(iii) $\Rightarrow$ (ii): Basta ver únicamente que para todo $a, b \in A$ existe $c \in A$ tal que $V(a) \cap V(b)=$ $V(c)$. Esto se debe a que $V(e) \cap V(f)=V(e+f-e f)$ en el caso en que $e, f \in A$ son idempotentes. La inclusión $V(e) \cap V(f) \subseteq V(e+f-e f)$ es evidente. Veamos la inclusión $V(e+f-e f) \subseteq V(e) \cap V(f)$ : sea $p \in V(e+f-e f)$, entonces $e+f-e f \in p$ y por tanto $e \cdot(e+f-e f) \in p$ y $f \cdot(e+f-e f) \in p$. Calculando uno se da cuenta de que $e \cdot(e+f-e f)=e^{2}+e f-e^{2} f=e+e f-e f=e$ y similarmente $f \cdot(e+f-e f)=$ $f e+f^{2}-e f^{2}=f e+f-e f=f$; por lo que $e, f \in p$ y por tanto $p \in V(e) \cap V(f)$.

(ii) $\Rightarrow$ (i): Gracias a la proposición 3.8 se tiene que $A$ es isomorfo a $\Gamma(\mathfrak{m}(A), \mathbf{X}(A))$ que es separable por el argumento del párrafo que antecede esta proposición.

La propiedad sobre la existencia de los idempotentes en la proposición 3.10 se relaciona con los anillos que a continuación describimos. Un anillo $A$ es de Baer si para todo ideal $I$ de $A$ existe un idempotente $e \in A$ tal que $(0: I)=\operatorname{Ann}(I)=(e)$. Se dice además que un anillo $A$ es de Baer débil si para todo $a \in A$ existe un idempotente $e \in A$ tal que $(0: a)=\operatorname{Ann}(a)=(e)$. Claramente se tiene que todo anillo de Baer es un anillo de Baer débil. En [8], el autor corrige la demostración de un resultado de [4], que establece que un anillo $A$ es de Baer si y solo si las componentes irreducibles de $\operatorname{Spec}(A)$ son $\operatorname{disjuntas}$ y $\mathfrak{m}(A)$ es un espacio de Stone. Otro resultado en este sentido es de [6] y establece que un anillo $A$ es de Baer débil si y solo si existe una retracción continua de $\operatorname{Spec}(A)$ en $\mathfrak{m}(A)$. En [8] se menciona el hecho de que la compacidad de $\mathfrak{m}(A)$ para un anillo de $A$ de Baer débil se obtiene por el resultado de [6].

El teorema 2.7 de [3] nos dice que si $A$ es un anillo reducido entonces:

$$
\operatorname{Ann}(S)=\bigcap\{p: p \in V(\operatorname{Ann}(S))\}
$$

para cualquier parte $S$ de $A$. Entonces:

$$
\operatorname{Ann}(a)=\bigcap\{p: p \in V(\operatorname{Ann}(a))\}=\bigcap\{p: p \notin V(a)\}=\bigcap\{p: a \notin p\}=\bigcap_{a \notin p} p .
$$

Ya estamos en medida de enunciar y probar el ligamen entre nuestra condición sobre los idempotentes y los anillos de tipo Baer:

Proposición 3.11 Sea $A$ un anillo reducido con unidad. Entonces $A$ es un anillo de Baer débil si y solo si para todo $a \in A$, existe un idempotente $e \in A$ tal que $V(a)=V(e)$.

Demostración: $(\Rightarrow)$ Sea $a \in A$ cualquiera. Por la condición de Baer débil se tiene un idempotente $e \in A$ tal que $\operatorname{Ann}(a)=(e)$. Entonces $V(\operatorname{Ann}(a))=V((e))=V(e)$. Como 
$A$ es reducido entonces $\mathfrak{m}(A) \backslash V(a)=V(e)$ y por tanto $V(a)=\mathfrak{m}(A) \backslash V(e)=V(f)$ en donde $f=1-e \in A$ que sigue siendo un idempotente.

$(\Leftarrow)$ Sea $a \in A$ arbitrario. Entonces existe $e \in A$ un idempotente tal que $V(a)=V(e)$. Entonces $\mathfrak{m}(A) \backslash V(a)=\mathfrak{m}(A) \backslash V(e)$, es decir que $V(\operatorname{Ann}(a))=V(\operatorname{Ann}(e))$. Entonces:

$$
\operatorname{Ann}(a)=\bigcap\{p: p \in V(\operatorname{Ann}(a))\}=\bigcap\{p: p \in V(\operatorname{Ann}(e))\}=\operatorname{Ann}(e) .
$$

Ahora poniendo $f=1-e \in A$ que también es un idempotente, veamos que $\operatorname{Ann}(e)=(f)$. Sea $x \in A$ tal que $x \in \operatorname{Ann}(e)$. Entonces $x \cdot e=0$ y por tanto $x=x \cdot 1=x \cdot(e+f)=$ $x \cdot e+x \cdot f=0+x \cdot f=x \cdot f$. Esto muestra que $x \in(f)$. Sea ahora $x \in(f)$, entonces $x=\alpha \cdot f$ para algún $\alpha \in A$. Por tanto $x \cdot e=(\alpha f) e=\alpha(f e)=\alpha \cdot 0=0$. Entonces $x \in \operatorname{Ann}(e)$. Esto prueba finalmente que $\operatorname{Ann}(a)=(f)$.

La proposición 3.10 junto con el resultado de la proposición 3.11 nos permite probar:

Teorema 3.12 Sea $A$ un anillo reducido y con unidad. Entonces son $A$ es un anillo separable si y solo si $A$ es un anillo de Baer débil.

Demostración: $(\Rightarrow)$ Sea $A$ un anillo separable. Entonces por la proposición 3.10 se tiene que para todo $a \in A$ existe $e \in A$ un idempotente tal que $V(a)=V(e)$. Por la proposición 3.11 se deduce que $A$ es un anillo de Baer débil.

$(\Leftarrow)$ Si $A$ es un anillo de Baer débil. Entonces se sabe que $\mathfrak{m}(A)$ es compacto. Por la proposición 3.11 se tiene que para todo $a \in A$, existe $e \in A$ un idempotente tal que $V(a)=$ $V(e)$. Entonces se satisface la condición (ii) de la proposición 3.10 que es equivalente a que el anillo $A$ sea separable.

Lo que hemos visto entonces es que la clase de anillos separables o de PP-anillos coincide también con la clase de anillos de Baer débil. Se aprecia también por la proposición 3.10, que esta clase es una subclase de los anillos de Henriksen-Jerison. El autor desconoce si esta subclase es impropia o si por el contrario todo anillo de Henriksen-Jerison es separable o de Baer débil o PP-anillo.

\section{Referencias}

[1] Chang, C.C.; Keisler, H.J. (1978) Model Theory. North-Holland, Amsterdam.

[2] Guier, J.I. (2001) "Boolean products of real closed valuation rings and fields", Annals of Pure and Applied Logic 112: 119-150.

[3] Henriksen, M.; Jerison, M. (1965) "The space of minimal prime ideals of a commutative ring", Trans. Amer. Math. Soc. 115: 110-130.

[4] Höchster, M. (1970) "Totally integrally closed rings and extremal spaces", Pacific J. Math. 32: 767-779. 
[5] Keimel, K. (1971) The Representation of Lattice-Ordered Groups and Rings by Sections of Sheaves. Lectures Notes in Mathematics 248, Springer-Verlag, Berlin Heidelberg: $1-98$.

[6] Kist, J. (1974) "Two characterizations for conmutative Baer rings", Pacific J. Math. 50: $125-134$.

[7] Ewad Abu Osba; Al-Ezeh, H. (2004) "The pure part of the ideals in $C(X)$ ", preprint.

[8] Picavet, G. (1980) "Ultrafiltres sur un espace spectral - Anneaux de Baer - Anneaux a spectre minimal compact", Math. Scand. 46: 23-53.

[9] Weispfenning, V. (1973) "Model-completeness and elimination of quantifiers for subdirect products of structures", Journal of Algebra 36: 252-277. 